\title{
ABSCHLUSSBERICHT
}

\section{ZWISCHENEVALUIERUNG \\ DER AKTIONSLINIE \\ „FEASIBILITY STUDIES“}

Alexander Keßler ${ }^{1}$ \& Dietmar Rößl ${ }^{2}$

25. August 2003

1 Mag. Dr. Alexander Keßler, Universitätsassistent am Institut für Betriebswirtschaftslehre der Kleinund Mittelbetriebe, alexander.kessler@wu-wien.ac.at

2 Univ-Doz. Mag. Dr. Dietmar Rößl, ao.Universitätsprofessor am Institut für Betriebswirtschaftslehre der Klein- und Mittelbetriebe, dietmar.roessl@wu-wien.ac.at

beide: Wirtschaftsuniversität Wien, Augasse 2-6, A-1090 Wien, Tel. 31 336-4592, Fax. 31 336-715 


\section{Präambel}

\section{Identifikation der Analysebereiche}

Ziel dieser Studie war es, die Aktionslinie „Feasibility Studies“ hinsichtlich ihrer Zielerreichung zu evaluieren. Die impliziten und expliziten Ziele wurden aus den zur Verfügung gestellten Unterlagen (Informationsfolder zur Aktionslinie, Protokoll des Programmkomitees, Aufgabenformulierung zur Zwischenevaluierung) abgeleitet und bei einem Meeting mit dem FFF hinterfragt und verifiziert.

Daneben sollen im Rahmen deskriptiver Auswertungen durch den FFF als relevant bezeichnete Daten analysiert werden (Aufgabenformulierung zur Zwischenevaluierung und ergänzende Interpretation durch den FFF).

Das Ergebnis dieses Arbeitsschrittes ist einerseits im „Projektdesign Evaluierung Aktionslinie Feasibility Studies Version 2" (siehe Anhang) dokumentiert, andererseits liegt es der Gliederung dieses Berichts zugrunde.

\section{Datenmaterial}

In die Auswertung wurden 183 Anträge einbezogen, das sind alle Anträge, die bis Ende Juni 2003 seitens des FFF entschieden wurden. Vorerst wurden 185 und später zwei weitere Datensätze zur Verfügung gestellt. Von diesen wurden zwei Anträge, die zurückgezogen wurden, und zwei Anträge, bei denen die Beschlussfassung seitens des FFF noch aussteht, eliminiert, womit sich die erwähnten 183 Datensätze ergeben, die diesem Bericht zugrunde liegen. Das Datenmaterial, das hier zur Auswertung gelangt, setzt sich aus folgenden Elementen zusammen:

- Datenbank (Antrag, technische und wirtschaftliche Stellungnahme, technischer und wirtschaftlicher Endbericht, Stammdaten)

- Fragebögen (Abschlussbericht) und

- im Falle von Folgeprojekten der Antrag sowie die technische und wirtschaftliche Stellungnahme

\begin{tabular}{|c|c|c|c|c|}
\hline \multirow[b]{2}{*}{$\begin{array}{l}\text { Daten- } \\
\text { umfang }\end{array}$} & \multicolumn{4}{|c|}{ vorhandenes Datenmaterial } \\
\hline & $\begin{array}{c}\text { Fragebögen / } \\
\text { Abschluss- } \\
\text { bericht }\end{array}$ & $\begin{array}{c}\text { Datenbank } \\
\text { (Umfang je nach } \\
\text { Fortschritt der FS) }\end{array}$ & \multicolumn{2}{|c|}{$\begin{array}{l}\text { technische und wirtschaftliche } \\
\text { Stellungnahme Folgeprojekte }\end{array}$} \\
\hline \multirow{5}{*}{183 Fälle } & \multirow{3}{*}{$\begin{array}{c}86 \text { mit } \\
\text { Abschluss- } \\
\text { bericht }\end{array}$} & 58 abgelehnt & & \\
\hline & & \multirow{4}{*}{125 gefördert } & \multirow{3}{*}{$\begin{array}{l}20 \text { Folge- } \\
\text { projekte }\end{array}$} & 15 gefördert \\
\hline & & & & 2 abgelehnt \\
\hline & \multirow{2}{*}{$\begin{array}{l}97 \text { (noch) ohne } \\
\text { Abschluss- } \\
\text { bericht }\end{array}$} & & & 3 offen \\
\hline & & & $\begin{array}{c}105 \text { keine bzw. } \\
\text { noch keine } \\
\text { Folgeprojekte }\end{array}$ & \\
\hline
\end{tabular}

Tabelle 1 
Zur Vereinfachung der Darlegungen werden folgende Abkürzungen bzw. Begriffsbildungen verwendet:
AFS
Aktionslinie „Feasibility Studies“
EFS
Ersteller der Feasibility Study
FB Fr. $n$
Fragennummer aus dem Fragebogen (Abschlussbericht)
Folgeprojekt beim FFF zur Förderung beantragtes Projekt, das auf der
FFF Feasibility Studie aufbaut
FS Forschungsförderungsfonds
MA Feasibility Study
Stammkunde Mitarbeiter
Neukunde
Antragsteller im Rahmen der AFS, der schon vorher FFF-Projekte beantragt hat
Antragsteller im Rahmen der AFS, der zum ersten mal ein FFF-Projekt beantragt 


\section{DESKRIPTIVE ANALYSE DER AKTIONSLINIE „FEASIBILITY STUDIES“}

\section{Analyse der geförderten Unternehmen}

\begin{tabular}{|c|c|c|}
\hline & geförderte Anträge & $\begin{array}{l}\text { nicht geförderte } \\
\text { Anträge }\end{array}$ \\
\hline Größe in MA ( $\varnothing 3$ Jahre) & $\varnothing 54 \mathrm{MA}$ & $\varnothing 50 \mathrm{MA}$ \\
\hline $\begin{array}{r}0-10 \mathrm{MA} \\
10-50 \mathrm{MA} \\
>50 \mathrm{MA}\end{array}$ & $\begin{array}{c}\text { \%-Anteil } \\
\\
30 \% \\
36 \% \\
34 \% \\
\end{array}$ & 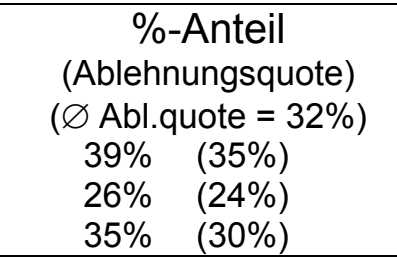 \\
\hline$\varnothing$ Größe nach Umsatz ( $\varnothing 3$ Jahre) & $\varnothing 7,900.000$ & $\varnothing 8,900.000$ \\
\hline $\begin{array}{l}\varnothing \text { Beschäftigtenwachstum (vom 3. zum } \\
\text { 1. Jahr vor Antragstellung) }\end{array}$ & $+4,5 \mathrm{MA}$ & $+10,5 \mathrm{MA}$ \\
\hline $\begin{array}{l}\varnothing \text { Umsatzwachstum (vom 3. zum 1. Jahr } \\
\text { vor Antragstellung) }\end{array}$ & $+1,600.000$ & $+4,200.000$ \\
\hline$\varnothing$ CashFlow ( $\varnothing 3$ Jahre) & $\varnothing 475.000$ & $\varnothing 655.000$ \\
\hline$\varnothing$ F\&E-Aufwand ( $\varnothing 3$ Jahre) & $\varnothing 215.000$ & $\varnothing 185.000$ \\
\hline$\varnothing$ F\&E-MA ( $\varnothing 3$ Jahre) & $\varnothing 2,8$ & $\varnothing 3,4$ \\
\hline $\begin{array}{r}0 \text { Jahre } \\
1-3 \text { Jahre } \\
4-10 \text { Jahre } \\
11-25 \text { Jahre } \\
\text { älter }\end{array}$ & $\begin{array}{c}\text { Verteilung der geför- } \\
\text { derten Anträge in \% } \\
7 \% \\
12 \% \\
23 \% \\
29 \% \\
29 \% \\
\end{array}$ & 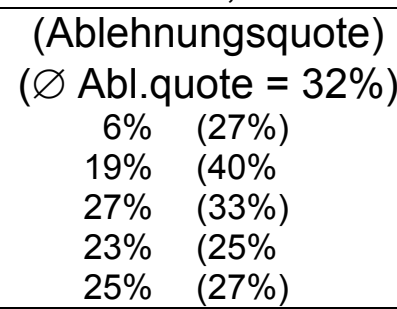 \\
\hline $\begin{array}{l}\text { 1er PLZ } \\
\text { 2er PLZ } \\
\text { 3er PLZ } \\
\text { 4er PLZ } \\
\text { 5er PLZ } \\
\text { 6er PLZ } \\
\text { 7er PLZ } \\
\text { 8er PLZ } \\
\text { 9er PLZ }\end{array}$ & $\begin{array}{c}15 \\
19 \\
10 \\
32 \\
6 \\
12 \\
0 \\
24 \\
7\end{array}$ & \begin{tabular}{ll}
\multicolumn{1}{c}{ Anzahl } \\
(Ablehnungsquote ) \\
( $\varnothing$ Abl.quote = 32\%) \\
11 & $(42 \%)$ \\
5 & $(21 \%)$ \\
4 & $(29 \%)$ \\
12 & $(27 \%)$ \\
& $(0 \%)$ \\
7 & $(37 \%)$ \\
1 & $(100 \%)$ \\
16 & $(40 \%)$ \\
2 & $(22 \%)$
\end{tabular} \\
\hline $\begin{array}{l}\text { Branchenverteilung der Projekt- } \\
\text { anträge (ÖNACE) } \\
\text { 15-er „Nahrungs- und Genussmittel“ } \\
\text { 20-er "Be- und Verarbeitung von Holz" } \\
\text { 24-er "Chemikalien und chem. Erzeugnisse" } \\
\text { 25-er "Gummi und Kunststoffwaren“ } \\
\text { 26-er "Glas, Waren aus Steinen und Erden } \\
\text { 29-er "Maschinenbau“ } \\
\text { 33-er "Medizin-, Mess-, Steuer- u. } \\
\text { Regelungstechnik, Optik“ } \\
\text { 45-er "Bauwesen“ } \\
\text { 72-er "Datenverarbeitung und Datenbanken“ } \\
\text { sonstige }\end{array}$ & $\begin{array}{c}16 \\
12 \\
6 \\
13 \\
7 \\
16 \\
\\
8 \\
8 \\
2 \\
37\end{array}$ & $\begin{array}{c}\text { Anzahl } \\
\text { (Ablehnungsquote ) } \\
\text { ( } \varnothing \text { Abl.quote = 32\%) } \\
2(11 \%) \\
7(37 \%) \\
2(25 \%) \\
3(19 \%) \\
6(46 \%) \\
8(33 \%) \\
7(47 \%) \\
2(20 \%) \\
7(78 \%) \\
14(27 \%)\end{array}$ \\
\hline
\end{tabular}


Bezogen auf die Unternehmensgröße zeigt sich, dass größere Unternehmen keine besseren Förderungschancen als kleinere Unternehmen haben.

Gründungsunternehmen und ältere Unternehmen haben im Vergleich zu Jungunternehmen (1-3 Jahre alt) etwas höhere Förderungschancen.

Weit überdurchschnittlich ist die Ablehnungsquote bei „Datenverarbeitung und Datenbanken“, „Medizin-, Mess-, Steuer- u. Regelungstechnik, Optik“ sowie bei „Gummi und Kunststoffwaren“.

\section{Zeit bis Folgeprojekt}

Hier interessiert die Frage, wie rasch nach einer Feasibility Studie ein Folgeprojekt beantragt wird.

Da die Unternehmen teilweise offenbar schon vor Verschriftlichung der Feasibility vom EFS bezüglich der Ergebnisse der FS informiert werden, kommt es vor, dass Unternehmen das Folgeprojekt bereits beantragen, noch bevor sie den Endbericht an den FFF weiterleiten. Daher musste hier auf die Zeitdifferenz Antragstellung AFS und Antragstellung Folgeprojekt abgestellt werden.

Im Durchschnitt wird der Antrag zum Folgeprojekt etwa ein Jahr (353 Tage) nach dem Antrag zur AFS gestellt.

- $20 \%$ der Folgeanträge werden innerhalb von 6 Monaten,

- 50\% werden zwischen 6 Monaten und einem Jahr und

- $30 \%$ der Folgeanträge werden später gestellt.

\section{Analyse der Ablehnungsgründe}

\begin{tabular}{|l|c||l|c|}
\hline Grund & Häufigkeit & Grund & Häufigkeit \\
\hline $\begin{array}{l}\text { keine Feasibility (Design, } \\
\text { Umsetzung, Expertise, etc.) }\end{array}$ & $16(28 \%)$ & $\begin{array}{l}\text { negative Bewertung der } \\
\text { Firma }\end{array}$ & $4(7 \%)$ \\
\hline $\begin{array}{l}\text { wirtschaftliche Frage im } \\
\text { Vordergrund (Marktstudie, etc.) }\end{array}$ & $9(16 \%)$ & $\begin{array}{l}\text { EFS nicht geeignet, } \\
\text { mangelndes Know-how }\end{array}$ & $3(5 \%)$ \\
\hline keine technische Innovation & $8(14 \%)$ & $\begin{array}{l}\text { Naheverhältnis zum EFS, } \\
\text { Konstellation zwischen } \\
\text { Antragsteller und EFS }\end{array}$ & $2(3 \%)$ \\
\hline kein KMU & $8(14 \%)$ & mangelnde Marktchancen & $2(3 \%)$ \\
\hline $\begin{array}{l}\text { andere „formelle“ Gründe } \\
\text { (Studie schon fertig,etc.) }\end{array}$ & $6(10 \%)$ & & \\
\hline
\end{tabular}

Tabelle 3

Offenbar kämpft die AFS damit, dass den Antragstellern oft nicht klar ist, was unter einer technischen Feasibility Studie gemeint ist, schließlich können die drei häufigsten Ablehnungsgründe auf Missverständnisse zurückgeführt werden (= $58 \%$ ). 


\section{Vergleich der weitergeführten}

\section{Anträge mit den nicht weitergeführten Anträgen}

Hier werden jene Anträge, die ein Folgeprojekt nach sich gezogen haben, mit jenen verglichen, die ohne Folgeprojekt geblieben sind. Da Folgeprojekte im Durchschnitt etwa ein Jahr nach dem ursprünglichen Antrag zur AFS beantragt werden, wurden lediglich jene Fälle, in denen zeitlich gesehen eine realistische Chance zur Beantragung eines Folgeprojekts bestand (vor dem 30.06.2002 eingereichte Feasibilities, um dem durchschnittlichen zeitlichen Abstand von 353 Tagen zwischen Feasibility und Folgeprojekt Rechnung zu tragen), untersucht. Insgesamt gingen in diesen Vergleich 87 vor dem 30.06.2002 beantragte und in weiterer Folge bewilligte Feasibility-Projekte ein, von denen bis zum Zeitpunkt der vorliegenden Evaluierung 18 zu Folgeprojektanträgen führten (14 gefördert, 2 offen, 2 abgelehnt) und 69 bisher ohne Folgeprojektantrag blieben. Die folgende Tabelle stellt die Unterschiede zwischen diesen beiden Gruppen in Bezug auf Unternehmenscharakteristika sowie auf die Bewertung der AFS auf Basis des Fragebogens im Schlussbericht dar:

\begin{tabular}{|c|c|c|c|}
\hline Kriterium & $\begin{array}{l}\text { Anträge vol } \\
\text { ohne Folge- } \\
\text { projekt }(n=69)\end{array}$ & $\begin{array}{l}30.06 .2002 \\
\text { mit Folge- } \\
\text { projekt }(n=18)\end{array}$ & $\begin{array}{l}\text { Anmer- } \\
\text { kungen }\end{array}$ \\
\hline $\begin{array}{l}\varnothing \text { Personalstand in den } 3 \text { Jahren vor } \\
\text { Antragseingang AFS }\end{array}$ & $60,7 \mathrm{MA}$ & $30,6 \mathrm{MA}$ & \\
\hline $\begin{array}{l}\varnothing \text { Personalstand F\&E in den } 3 \mathrm{~J} . \text { vor } \\
\text { Antragseingang AFS }\end{array}$ & $2,7 \mathrm{MA}$ & 1,6 MA & \\
\hline $\begin{array}{l}\varnothing \text { Umsatz in den } 3 \text { Jahren vor } \\
\text { Antragseingang AFS }\end{array}$ & $€ 9,110.000,-$ & $€ 4,698.000,-$ & \\
\hline $\begin{array}{l}\varnothing \text { Cash Flow in den } 3 \text { Jahren vor } \\
\text { Antragseingang AFS }\end{array}$ & $€ 645.000,-$ & $€ 223.000,-$ & $\begin{array}{l}(\text { Sig **) } \\
(t=2,55 ; \\
p=0,01)\end{array}$ \\
\hline $\begin{array}{l}\varnothing \text { F\&E Aufwand in den } 3 \text { Jahren vor } \\
\text { Antragseingang AFS }\end{array}$ & $€ 157.000,-$ & $€ 225.000,-$ & \\
\hline $\begin{array}{l}\text { Alter des Unternehmens zum } \\
\text { Antragszeitpunkt AFS }\end{array}$ & $\varnothing 25,4$ Jahre & $\varnothing 9,3$ Jahre & $\begin{array}{l}\text { (Sig. } * * * *) \\
(\mathrm{t}=4,01 ; \\
\mathrm{p}=0,00)\end{array}$ \\
\hline Höhe des Förderungsbeitrages AFS & $\varnothing € 7.340,-$ & $\varnothing € 10.600,-$ & \\
\hline $\begin{array}{l}\text { Eignung der Feasibility als } \\
\text { Entscheidungsgrundlage (FB Fr. 2) }\end{array}$ & $\varnothing 1,32$ & $\varnothing 1,18$ & \\
\hline $\begin{array}{l}\text { Ergebnis auch anders erreichbar (FB } \\
\text { Fr. 3) }\end{array}$ & $\varnothing 3,39$ & $\varnothing 3,24$ & \\
\hline $\begin{array}{l}\text { gutes Preis-/Leistungsverhältnis (FB } \\
\text { Fr. 4) }\end{array}$ & $\varnothing 1,74$ & $\varnothing 2,24$ & $\begin{array}{l}\text { (Sig. **) } \\
(\mathrm{t}=-2,61 ; \\
\mathrm{p}=0,01)\end{array}$ \\
\hline $\begin{array}{l}\text { Professionalität/Qualität der } \\
\text { Recherche (FB Fr. 5) }\end{array}$ & $\varnothing 1,27$ & $\varnothing 1,76$ & $\begin{array}{l}\text { (Sig. a) } \\
(t=-1,98 \\
p=0,06)\end{array}$ \\
\hline $\begin{array}{l}\text { Ergebnis entspricht den Erwartungen } \\
\text { (FB Fr. 6) }\end{array}$ & $\varnothing 1,71$ & $\varnothing 1,65$ & \\
\hline
\end{tabular}




\begin{tabular}{|c|c|c|c|}
\hline $\begin{array}{l}\text { weiterführendes Projekt beabsichtigt } \\
\text { (FB Fr. 7) }\end{array}$ & $\varnothing 1,59$ & $\varnothing 1,24$ & $\begin{array}{l}\text { (Sig. a) } \\
(t=1,68 \\
p=0,10)\end{array}$ \\
\hline $\begin{array}{l}\text { weitere Kooperation mit Ersteller } \\
\text { Feasibility (FB Fr. 8) }\end{array}$ & $\varnothing 1,29$ & $\varnothing 1,41$ & \\
\hline $\begin{array}{l}\text { Beauftragung einer neuerlichen } \\
\text { Feasibility (FB Fr. 9) }\end{array}$ & $\varnothing 1,18$ & $\varnothing 1,06$ & \\
\hline $\begin{array}{l}\text { Zufriedenheit mit der Abwicklung } \\
\text { durch FFF (FB Fr. 10) }\end{array}$ & $\varnothing 1,13$ & $\varnothing 1,19$ & \\
\hline \multicolumn{4}{|c|}{$\begin{array}{l}\text { Anmerkungen: } \\
\text { Die Antworten zu den Fragen des Fragebogens wurden auf einer } 4 \text {-stufigen Skala codiert, } 1=\text { sehr gut } \\
\text { bzw. ja, ...4=sehr schlecht bzw. nein. }\end{array}$} \\
\hline $\begin{array}{l}\text { Signifikanznivaus: } \\
\text { a...Irtumswahrscheinlichkeit zwischen } 5-10 \% \\
\text { *... Irrtumswahrscheinlichkeit zwischen } 1-5 \% \\
\text { **...Irrtumswahrscheinlichkeit zwischen } 0,1 \\
\text { *** Irrtumswahrscheinlichkeit < 0,1\% }\end{array}$ & $\%$ & & \\
\hline
\end{tabular}

\section{Tabelle 4}

Jene Unternehmen, die schließlich aus der FS ein Folgeprojekt beantragten, sehen das Preis-/Leistungsverhältnis kritischer. Es sind die kleineren Unternehmen, für die die Kosten trotz Förderung schwer zu tragen sind. Das zeigen auch Hinweise auf den Fragebögen, wie z.B. „für ein KMU teuer, aber sonst gar nicht finanzierbar"

Interessant - weil wohl überraschend - ist, dass die Professionalität gerade von jenen Unternehmen, die schließlich die FS weitergeführt haben, kritischer gesehen wird. (Wir vermuten, dass diese Unternehmen schon bei Antragstellung konkretere Vorstellungen von dem hatten, was sie für ihre Entscheidungen benötigten; während in der anderen Antragsgruppe auch wenig überlegte „Versuchsballons“ stecken könnten.)

Folgeprojekte wurden öfter von kleineren und signifikant öfter von jüngeren Unternehmen in Angriff genommen.

Die Wahrscheinlichkeit, dass eine FS ein Folgeprojekte nach sich zieht, steigt auch deutlich (aufgrund der hohen Standardabweichung und der kleinen Fallzahl kann allerdings keine Signifikanz nachgewiesen werden) mit der Höhe des Förderungsbeitrages. D.h., teure FS haben in Bezug auf Umsetzung eine höhere Effektivität. 


\section{MODUL I: EFFEKTIVITÄT DER AKTIONSLINIE „FEASIBILITY STUDIES“"}

\section{Erschließung eines professionellen Beratungspotentials}

Die gesprächsweise angedeutete Befürchtung bei Lancierung der AFS, dass hier nur eine Handvoll etablierter Institute „zum Zug kommen könnten“, hat sich als haltlos erwiesen. Es konnte ein breites Beratungspotential für die antragstellenden KMUs mobilisiert werden:

Die FS der 125 geförderten Anträge wurden von 93 verschiedenen Instituten bzw. Abteilungen durchgeführt, fasst man die einzelnen Abteilungen von ofi zusammen verbleiben nach wie vor 85 unterschiedliche EFS (aufgrund der Autonomie von Universitätsinstituten wurden diese einzeln gezählt). Somit kam es zum erwünschten Effekt, mit dieser Aktionslinie nicht nur die Unternehmen zu fördern, sondern die österreichische Forschungslandschaft generell anzustoßen.

\begin{tabular}{|c|c|c|c|c|c|c|c|}
\hline \multirow[b]{2}{*}{ EFS } & \multicolumn{2}{|c|}{$\begin{array}{c}\text { alle } \\
\text { Anträge }\end{array}$} & \multicolumn{2}{|c|}{$\begin{array}{l}\text { geför- } \\
\text { derte } \\
\text { Anträge }\end{array}$} & \multicolumn{3}{|c|}{$\begin{array}{l}\text { Erfolgsquote (Anteil der Folgeprojekte) } \\
\text { hier werden aufgrund der durchschnittlichen Zeitdauer } \\
\text { bis zur Beantragung des Folgeprojekts nur die } \\
\text { Anträge vor } 30.06 .2002 \text { berücksichtigt }\end{array}$} \\
\hline & $n$ & $\%$ & $\mathrm{n}$ & $\%$ & $\begin{array}{c}n \text { vor } \\
30.06 .02\end{array}$ & $\begin{array}{c}\text { davon mit } \\
\text { Folgeprojekt } \\
\text { (Erfolgsquote) }\end{array}$ & $\begin{array}{c}\text { davon mit } \\
\text { gefördertem bzw. }(+) \\
\text { offenem Folgeprojekt }\end{array}$ \\
\hline Universitätsinstitute & 51 & $30 \%$ & 37 & $30 \%$ & 25 & $7(28 \%)$ & $5+1(20 \%$ bzw. $24 \%)$ \\
\hline andere private Ersteller & 38 & $23 \%$ & 28 & $23 \%$ & 15 & $(33 \%)$ & $\begin{array}{ll}4 & (27 \%)\end{array}$ \\
\hline ofi & 26 & $16 \%$ & 20 & $16 \%$ & 16 & $(19 \%)$ & $2+1(13 \%$ bzw. 19\%) \\
\hline ARP & 12 & $7 \%$ & 9 & $7 \%$ & 7 & $(29 \%)$ & $2 \quad(29 \%)$ \\
\hline Seibersdorf & 10 & $6 \%$ & 6 & $5 \%$ & 4 & $(0 \%)$ & $(0 \%)$ \\
\hline $\mathrm{FH} / \mathrm{HTL}$ & 8 & $5 \%$ & 6 & $5 \%$ & 3 & $(0 \%)$ & $(0 \%)$ \\
\hline bautechn. Institut Linz & 7 & $4 \%$ & 6 & $5 \%$ & 6 & $(0 \%)$ & $(0 \%)$ \\
\hline Joanneum & 6 & $4 \%$ & 3 & $2 \%$ & 2 & $(50 \%)$ & $(50 \%)$ \\
\hline Holzforschung Austria & 6 & $4 \%$ & 5 & $4 \%$ & 5 & $(0 \%)$ & $(0 \%)$ \\
\hline LVA Wien & 4 & $2 \%$ & 4 & $3 \%$ & 4 & $(0 \%)$ & $(0 \%)$ \\
\hline keine Angabe & 15 & & 1 & & 0 & 0 & 0 \\
\hline
\end{tabular}

Die „Erfolgsquoten“ können nicht direkt verglichen werden; sowohl die geringen Fallzahlen als auch die unterschiedlichen technischen Bereiche der EFS sprechen gegen eine solche Interpretation. Aufgrund der Zufriedenheit mit dem EFS kann darauf geschlossen werden, dass im wesentlichen auch der „passende“ EFS ausgewählt wurde:

\begin{tabular}{|l|c|c|c|c|}
\hline EFS & $\begin{array}{c}\text { n geförderte und } \\
\text { abgeschlossene } \\
\text { Projekte (FB } \\
\text { vorhanden) }\end{array}$ & $\begin{array}{c}\text { weitere } \\
\text { Kooperation mit } \\
\text { EFS (FB Fr.8) } \\
1=j a ~ \ldots 4=\text { nein }\end{array}$ & $\begin{array}{c}\text { Preis-/Leistungs- } \\
\text { verhältnis (FB Fr. 4) } \\
1=\text { sehr gut ... } \\
4=\text { sehr schlecht }\end{array}$ & $\begin{array}{c}\text { Professionaliät / Quali- } \\
\text { tät der FS (FB Fr.5) } \\
1=\text { sehr gut ... } \\
4=\text { sehr schlecht }\end{array}$ \\
\hline Universitätsinstitute & 22 & 1,4 & 1,6 & 1,4 \\
\hline andere private EFS & 15 & 1,5 & 2,1 & 1,8 \\
\hline ofi & 16 & 1,2 & 1,9 & 1,3 \\
\hline ARP & 8 & 1 & 1,9 & 1,3 \\
\hline Seibersdorf & 5 & 2,2 & 1,8 & 1 \\
\hline FH / HTL & 4 & 1,5 & 1,8 & 1 \\
\hline bautechn. Institut Linz & 6 & 1,3 & 1,8 & 2 \\
\hline Joanneum & 2 & 2 & 2,5 & 1,8 \\
\hline Holzforschung Austria & 5 & 1,2 & 1,8 & 1,7 \\
\hline LVA Wien & 3 & 1,0 & 2,7 & \\
\hline \multicolumn{2}{|c|}{ grau sind jene Ergebnisse gesetzt, die auf wenigen Fällen beruhen und daher nicht aussagekräftig sind } \\
\hline
\end{tabular}

Tabelle 6 


\section{Verhaltensfraqe: Einstellunqsänderunqen qeqenüber externen Beratern}

Als zentrales Indiz für eine durch die AFS ausgelöste Einstellungsänderung gegenüber externen Beratern wurde das erstmalige Förderungswerben einer Unternehmung beim FFF im Rahmen der AFS definiert. Von den insgesamt 183 Anträgen, die dieser Evaluierung zu Grunde liegen, entfallen 91 Fälle (59 gefördert $=64,8 \% ; 32$ abgelehnt $=35,2 \%$ ) auf diese Gruppe der Neukunden, während 92 Anträge (66 gefördert $=71,7 \% ; 26$ abgelehnt $=28,3 \%$ ) von Stammkunden (bereits mind. ein Projektantrag beim FFF vor der AFS) kamen. Im Folgenden wird speziell die Gruppe der Neukunden dahingehend analysiert, inwieweit sie mit dem Ablauf der AFS zufrieden war und Interesse an weiterführenden Projekten bzw. neuerlichen FeasibilityStudien zeigt (Fragebogen Schlussbericht) bzw. ob sich im Weiteren tatsächlich Folgeanträge aus dieser Gruppe ergeben haben.

Aus dem von den Unternehmungen im Rahmen der Schlussberichterstattung ausgefüllten Fragebogen sind vor allem die folgenden Fragen von besonderer Relevanz in Bezug auf eine dauerhafte Einstellungsänderung gegenüber externen Beratern:

\begin{tabular}{|l|c|}
\hline Frage & $\begin{array}{c}\text { Mittelwert der Neukunden } \\
\text { (1=ja ... 4=nein) }\end{array}$ \\
\hline weiterführendes Projekt beabsichtigt (FB Fr. 7) & 1,59 \\
\hline weitere Kooperation mit EFS (FB Fr. 8) & 1,29 \\
\hline Beauftragung einer neuerlichen Feasibility (FB Fr. 9) & 1,18 \\
\hline
\end{tabular}

$$
\text { Tabelle } 7
$$

Die Meinungsäußerungen der Neukunden sowohl in Bezug die Beabsichtigung eines weiterführenden Projekts auf Basis der erstellten Feasibility-Studie und eine weitere Kooperation mit dem Ersteller der Studie als auch in Bezug auf die Beauftragung weiterer Feasibility-Studien im Falle neuerlicher Themen sind äußerst positiv, was als Indiz für eine dauerhafte Einstellungsänderung gewertet werden kann.

Um eine eventuelle Einstellungsänderung speziell in Bezug auf die Kosten für eine extern vergebene Feasibility-Studie zu hinterfragen, wurde auch der Frage 4 aus dem FB („Wie beurteilen Sie das Preis-/Leistungsverhältnis der Studie?“) ausgewertet. Hierbei ergab sich auf einer ebenfalls 4-stufigen Skala (von 1=sehr gut bis $4=$ sehr schlecht) ein Mittelwert von 1,89. Auch dies stellt unter der Prämisse, dass die Unternehmen extern vergebene Feasibility-Studien vorab als eher kostenintensiv einschätzten, ein positives Ergebnis im Sinne einer möglichen Einstellungsänderung dar.

Neben diesen subjektiven Einschätzungen der Neukunden zum Zeitpunkt der Schlussberichterstattung im Rahmen der AFS zeigten sich hinsichtlich der tatsächlichen Folgeanträge von Neu- und Stammkunden folgende Entwicklungen:

Von den 91 Anträgen von Neukunden im Rahmen der AFS mündeten 13 (14,3\%) in weiterführende FFF Projekte, von denen zum Zeitpunkt der Datenerfassung für diese Evaluierung 9 (69\%) bewilligt, 1 (8\%) abgelehnt und 3 (23\%) noch nicht entschieden waren. Diese Rate stellt verglichen mit 7 von 92 Projekten (7,6\%), die in der Gruppe der Stammkunden in weiterführende Projekte mündeten (6 bewilligt, 1 abgelehnt), ein deutlich (wenn auch nicht signifikant) höheres Ausmaß an Folgeprojekten dar.

Betrachtet man alle weiteren Projektanträge (nicht nur die Folgeprojekte der FS), die von den beiden Vergleichsgruppen beim FFF eingebracht wurden, so ergibt sich für 
die Gruppe der Stammkunden ein Mittelwert von 0,87 Projekten während sich für die Neukunden lediglich ein Mittelwert von 0,27 ergibt. Dieser signifikante Unterschied $(t=3,52 ; p=0,00)$ wird allerdings dadurch relativiert, dass es ausschließlich in der Gruppe der Stammkunden Unternehmungen gibt, die seit Antragseingang im Rahmen der AFS eine größere Zahl weiterer Projektanträge (bis zu 7) einbrachten, während der Maximalwert in der Gruppe der Neukunden bei zwei nachfolgenden Projektanträgen liegt. Darüber hinaus muss in Rechnung gestellt werden, dass sich die Einstellungsänderung bei den Neukunden zu Forschungsprojekten und zum FFF in einer schrittweisen - von der aktuellen FS ausgehenden - Änderung des Forschungsverhaltens zeigen wird. Dies zeigen auch die Zahlen: Neukunden beantragten $\varnothing 0,14$ Folgeprojekte und $\varnothing 0,13$ sonstige FFF-Projekte; Stammkunden hingegen nur $\varnothing 0,08$ Folgeprojekte aber $\varnothing 0,79$ sonstige FFF-Projekte.

\section{Erhöhung der Forschungsausgaben}

Zugang zu Neukunden (dazu siehe auch oben I./2)

Da man vor allem bei den "Neukunden“ argumentieren kann, dass diese ohne die AFS keine FS durchgeführt hätten, sind die bei dieser Gruppe induzierten Forschungsausgaben für die Effektivität der AFS von besonderer Relevanz.

Von den 91 von Neukunden beantragten Studien wurden 59 gefördert, was zu einem Gesamtförderungsbetrag seitens des FFF von $€ 428.089$ (Mittelwert $€ 7.256,-$ ) für diese Gruppe führte. In Summe mit den $€$ 183.467,- (Mittelwert $€$ 3.110,-) an Anteilen der jeweiligen Förderungswerber konnten somit direkte Forschungsausgaben in der Höhe von $€$ 611.556.- bei Unternehmen mobilisiert werden, die zuvor noch keine Anträge beim FFF gestellt hatten.

direkte Forschungsausgaben

\begin{tabular}{|c|c|c|c|c|c|}
\hline & \multicolumn{2}{|c|}{ beantragte Gesamtkosten } & $\begin{array}{l}\text { genehmigte } \\
\text { Kosten }\end{array}$ & $\begin{array}{l}\text { Förderung } \\
\text { FFF }(70 \%)\end{array}$ & \multirow{2}{*}{\begin{tabular}{|c|} 
Anteil \\
Förderungs- \\
werber $(30 \%)$
\end{tabular}} \\
\hline & $\begin{array}{l}\text { abgelehnt } \\
(n=58)\end{array}$ & \multicolumn{3}{|c|}{ gefördert $(n=125)$} & \\
\hline Mittelwerte & $€ 14.922,-$ & $€ 24.083,-$ & $€ 11.669,-$ & $€ 8.168,-$ & $€ 3.501,-$ \\
\hline Mediane & $€ 10.951,-$ & $€ 11.400,-$ & $€ 10.611,-$ & $€ 7.428,-$ & $€ 3.183,-$ \\
\hline Minimum & $€ 3.000,-$ & $€ 5.451,-$ & $€ 4.600,-$ & $€ 3.220,-$ & $€ 1.380,-$ \\
\hline Maximum & $€ 155.811,-$ & $€ 723.968,-$ & $€ 72.600,-$ & $€ 50.820,-$ & $€ 21.780,-$ \\
\hline Summen & $€ 865.447,-$ & $€ 3,010.414,-$ & $€ 1,458.656,-$ & $€ 1,021.059,-$ & $€ 437.597,-$ \\
\hline $\begin{array}{l}\text { Hier muss be } \\
24.083) \text { stark } \\
\text { den durchsch } \\
\text { ist auch durch }\end{array}$ & rgleich der & diane erkennk & 4 & h beantragten $\mathrm{G}$ & $\begin{array}{l}\text { Mittelwert (€ } \\
\text { samtkosten und } \\
\text { chein hat (dies }\end{array}$ \\
\hline
\end{tabular}

Tabelle 8

Durch die AFS wurden schließlich rund $€ 1,5$ Mio. für Feasibility Studien mobilisiert, davon etwa $€ 1$ Mio. öffentliche Mittel und $€$ 0,5 Mio. private Mittel. 
induzierte Forschungs- und Entwicklungsaufwendungen

Da Folgeprojekte im Durchschnitt ein Jahr nach dem Antrag im Rahmen der AFS beantragt werden, werden bei dieser Analyse die geförderten Anträge bis 30. Juni 2002 den bisher beantragten Folgeprojekten aus dieser Kohorte gegenübergestellt.

\begin{tabular}{|c|c|}
\hline AFS vor 30.6.02 & Folgeanträge \\
\hline 128 Anträge vor 30.06.02 & 18 Anträge in diesem Zeitraum \\
\hline $\begin{array}{l}\text { davon } 87 \text { gefördert } \\
\text { Die Folgeprojektquote liegt bei } 2 \\
\text { Die Quote der erfolgreichen } \\
\text { Entscheidung der offenen Anträg }\end{array}$ & $\begin{array}{l}\text { davon } 14 \text { gefördert, } 2 \text { noch offen, } 2 \text { abgelehnt } \\
0,5 \% \text {. } \\
\text { Folgeprojekte liegt in Abhängigkeit von der } \\
\text { e zwischen } 16 \%-18,5 \% \text {. }\end{array}$ \\
\hline $\begin{array}{l}\text { Diese haben } € 700.000 \text { an } \\
\text { Förderungen bezogen. }\end{array}$ & $\begin{array}{l}\text { Diese } 14 \text { Folgeprojekte bedeuten } \\
-5 \text { geschaffene F\&E-Arbeitsplätze } \\
\text { - } € 2,5 \text { Mio. induzierte Projektinvestionen } \\
\text { - davon } € 0,55 \text { Mio. induzierte Forschungs- } \\
\text { aufwendungen } \\
\text { Hochgerechnet auf } 16 \text { Folgeprojekte (inkl. der } \\
\text { beiden offenen Anträge) ergeben sich } \\
-5,7 \text { geschaffene F\&E-Arbeitsplätze } \\
-€ 2,9 \text { Mio. induzierte Projektinvestitionen } \\
- \text { davon } € 0,65 \text { Mio. induzierte Forschungs- } \\
\text { aufwendungen }\end{array}$ \\
\hline
\end{tabular}

Daraus ergibt sich ein Förderungsmultiplikator von 3,6-4,2 der AFS (ein $€$ Förderung der AFS induziert $€ 3,6-4,2$ Investitionen in Folgeprojekte). Dabei sind ohne FFFUnterstützung durchgeführte Folgeprojekte und Umsetzungen natürlich nicht enthalten!

\section{Vergrößerung der Basis innovierender Unternehmen}

Von den insgesamt 183 Anträgen, die dieser Evaluierung zu Grunde liegen, entfallen 91 Fälle (65\% gefördert, 35\% abgelehnt) auf Neukunden, während 92 Anträge (72\% gefördert; $28 \%$ abgelehnt) von Stammkunden (zuvor bereits mind. ein Projektantrag beim FFF vor der AFS) kamen.

Unterstellt man, dass die Neukunden durch die AFS zum ersten Mal eine technische Feasibility Studie beauftragt haben und damit zum ersten Mal ein Innovationsvorhaben umsetzen wollen oder zumindest zum ersten Mal ein solches Vorhaben professionell angehen wollen, kann man sagen, dass durch die AFS die Basis der innovierenden Unternehmen bzw. die Basis der professionell innovierenden Unternehmen vergrößert wurde.

Auf Basis der vorliegenden Daten kann diese Frage leider nicht fundierter analysiert werden (siehe Empfehlungen zur Verbesserung der Evaluierungsmöglichkeiten). 


\section{Gründerunternehmen ansprechen}

6,5\% der Anträge stammten von erst im Jahr der Antragstellung gegründeten Unternehmen. 20,5\% der Anträge stammten von Unternehmen, die zum Zeitpunkt der Antragstellung höchsten 3 Jahre alt waren. Das Ziel „Auch Gründungsunternehmen sollen angesprochen werden!" kann als erfüllt angesehen werden.

Auch wenn die Ablehnungsquote bei den Jungunternehmen etwas höher liegt, bleiben schließlich 23 geförderte Gründungs- bzw. Jungunternehmen $(=19 \%$ aller geförderten Unternehmen).

\begin{tabular}{|l|c|c|c|c|c|}
\hline Alter & 0 Jahre & 1-3 Jahre alt & 4-10 Jahre alt & 11-25 Jahre alt & $>$ 25 Jahre alt \\
\hline $\begin{array}{c}\text { Ablehnungsquote in } \% \\
\text { (Durchschnitt }=32 \% \text { ) }\end{array}$ & $27 \%$ & $40 \%$ & $33 \%$ & $25 \%$ & $27 \%$ \\
\hline
\end{tabular}

\section{Tabelle 10}

Die Gründungs- und Jungunternehmen beabsichtigen, das Projekt fortzusetzen, daher ist das Ansprechen dieser Gruppe in Bezug auf die Ziele der AFS besonders funktional:

\begin{tabular}{|c|c|c|c|c|c|}
\hline Alter & 0 Jahre & 1-3 Jahre alt & 4-10 Jahre alt & 11-25 Jahre alt & $>25$ Jahre alt \\
\hline $\begin{array}{l}\text { sichere Absicht, das } \\
\text { Projekt fortzusetzen in } \\
\% \text { (Durchschnitt }=65 \% \text { ) }\end{array}$ & $100 \%$ & $88 \%$ & $58 \%$ & $64 \%$ & $60 \%$ \\
\hline $\begin{array}{l}\text { sichere Absicht, das } \\
\text { Projekt fortzusetzen } \\
\left.\text { (Sign. Fisher, } \mathrm{X}^{2}: *\right)\end{array}$ & \multicolumn{2}{|c|}{$92 \%$} & \multicolumn{3}{|c|}{$61 \%$} \\
\hline
\end{tabular}

Tabelle 11

\section{6. objektive Ideenprüfung und gaf. Anreize zur Verwirklichung geben}

Ein Indiz der objektiven Prüfung ist die unter I./1. dargelegte starke Streuung der EFS: Die FS der 125 geförderten Anträge wurden von 93 verschiedenen Instituten bzw. Abteilungen durchgeführt (bei Zusammenfassung der einzelnen Abteilungen von ofi verbleiben nach wie vor 85). Das spricht für die Auswahl eines fachlich adäquaten EFS, womit eine fachlich fundierte Prüfung der Vorhaben gegeben erscheint.

Als weiterer Indikator für eine objektive Prüfung kann ins Treffen geführt werden, dass nur $1 \%$ der Anträge (= $3 \%$ der Ablehnungen) wegen einem Naheverhältnis zwischen Antragsteller und EFS abgelehnt wurden.

Schließlich soll die technische Bewertung - bzw. die dieser FFF-internen Bewertung zugrundeliegenden Ergebnisse der FS - als Anreiz für die Umsetzung herangezogen werden. Wenn die Qualität als auch die Verwertungschancen eines Projekts nach Vorliegen der FS hoch bewertet werden, kann vermutet werden, dass vom Ergebnis der FS eine Motivation zur Projektumsetzung ausgeht. Umgekehrt werden negative Ergebnisse der FS - womit geringe Bewertungen seitens des FFF korrelieren sollten - dem Antragsteller wohl als Warnungen (hohe technische Risken, problematische Verwertungschancen) dienen.

\begin{tabular}{|l|c|}
\cline { 2 - 2 } \multicolumn{1}{c|}{} & technische Bewertung (Qualität + Verwertung) \\
\hline Mittelwert & 35,7 \\
\hline Perzentile & $25 \% \leq 30,50 \% \leq 40,75 \% \leq 42$ \\
\hline
\end{tabular}

Tabelle 12 
Zwischen der technischen Bewertung der Ergebnisse der FS durch den FFF und der beabsichtigten Weiterführung des Projekts (FB Fr. 7) konnte ein schwach signifikanter Zusammenhang nachgewiesen werden:

\begin{tabular}{|l|c|c|}
\cline { 2 - 3 } \multicolumn{1}{c|}{} & $\begin{array}{l}\text { werden Projekt sicher oder } \\
\text { ziemlich sicher fortführen }\end{array}$ & $\begin{array}{l}\text { werden Projekt nicht oder } \\
\text { kaum weiterführen }\end{array}$ \\
\hline $\begin{array}{l}\text { Mittelwert der technischen } \\
\text { Endbewertung }\end{array}$ & $37,4 \quad$ & 25,4 \\
\hline
\end{tabular}

Tabelle 13

Zwischen der technischen Bewertung und der tatsächlichen Beantragung von weiterführenden Projekten konnte allerdings kein Zusammenhang nachgewiesen werden. Ob ein solcher Zusammenhang mit der Projektfortführung generell - d.h., Projektfortführung nicht nur mit, sondern auch ohne FFF-Förderungsantrag - vorliegt, kann nicht beantwortet werden, da keine Informationen über weiterführende Projekte ohne FFF-Förderungsantrag vorliegen.

Hingegen zeigt sich, dass abgelehnten Folgeprojekten eine wesentlich schwächere technische Bewertung des AFS-Antrages vorausgeht (22,5 versus 32,7 technische Bewertung des AFS-Antrages), was aber angesichts der kleinen Fallzahl nicht überinterpretiert werden soll.

\section{7. professioneller Start von Innovationsprojekten}

Die Erreichung des Zieles, Innovationsprojekten einen professionellen Start zu ermöglichen, wurde einerseits auf Basis von Einschätzungen der geförderten Unternehmen (Fragebogen Abschlussbericht) und andererseits auf Basis der Analyse von Ablehnungsgründen sowie der Qualität von eventuellen Folgeprojekten hinterfragt.

Im Rahmen der Analyse der Einschätzungen der geförderten Unternehmen erschienen vor allem die Fragen nach der Eignung der Studie als Entscheidungsgrundlage (FB Fr.2), der Möglichkeit des Erreichens des Ergebnisses auf anderem Wege (FB Fr. 3), die Professionalität und Qualität der Durchführung der Studie (FB Fr. 5) sowie des Einflusses der Ergebnisse auf den Innovationsprozess (kam es auch zu unerwarteten Ergebnissen - FB Fr. 6) relevant. Die folgende Tabelle zeigt die Ergebnisse der Auswertungen dieser Fragen:

\begin{tabular}{|c|c|}
\hline Frage & $\begin{array}{l}\text { Mittelwert (1="ja", 2="ehr ja", } \\
\text { 3="eher nein", 4="nein" bzw. } \\
\text { 1="sehr gut", 2="gut", } 3= \\
\text { "schlecht", 4="sehr schlecht") }\end{array}$ \\
\hline $\begin{array}{l}\text { Bietet die Studie eine ausreichende } \\
\text { Entscheidungsgrundlage? (FB Fr. 2) }\end{array}$ & 1,27 \\
\hline $\begin{array}{l}\text { Hätte das Ergebnis auch anders erreicht werden können? } \\
\text { (FB Fr. 3) }\end{array}$ & 3,38 \\
\hline Professionalität und Qualität der Durchführung (FB Fr. 5) & 1,38 \\
\hline Entspricht das Ergebnis Ihren Erwartungen? (FB Fr. 6) & 1,67 \\
\hline
\end{tabular}

Tabelle 14

Die Darstellung der Mittelwerte zeigt, dass die Feasibility-Studien zum überwiegenden Teil eine ausreichende Entscheidungsgrundlage darstellten (lediglich in 4 
Fällen wurde diese Frage mit „nein“ beantwortet), dass das Ergebnis nur schwer anders hätte erreicht werden können (nur 3 mal ,ja“) und dass auch die Qualität der Durchführung der Studien im Schnitt sehr gut beurteilt wurde (62 mal „sehr gut“, 17 mal "gut“, 5 mal „schlecht“, 2 mal „sehr schlecht“). Auf die Frage, ob das Ergebnis der Feasibility-Studien den Erwartungen der Unternehmungen entsprach, antworteten 56 mit „ja“, 9 mit „eher ja“, 14 mit „eher nein“ und 7 mit „nein“. Ob die „nein“ bzw. „eher nein" Antworten auf überraschend positive oder überraschend negative Ergebnisse der Feasibilities bzw. ob die „Ja-Antworten“ auf Missverständnisse (siehe „Empfehlungen zur Verbesserung der Evaluierungsmöglichkeiten der AFS“) zurückzuführen sind, kann auf Grundlage der vorliegenden Daten nicht beantwortet werden. Jedenfalls stellen überraschende Ergebnisse wichtige Entscheidungsgrundlagen für den Innovationsprozess dar, indem Unternehmen mit unrealistischen Ideen die Problematik in Bezug auf die Machbarkeit aufgezeigt wird sowie vorab von ihren (machbaren) Ideen wenig überzeugte Unternehmen in Richtung der Fortführung des Innovationsprozesses bestärkt werden.

In Beantwortung der Frage, inwieweit unrealistische Ideen im Rahmen der Bearbeitung der Förderungsansuchen bereits frühzeitig (vor Erstellung einer Feasibility) gestoppt wurden, werfen wir noch einmal einen Blick auf die Analyse der Ablehnungsgründe (vgl. Tabelle 3):

Aus der Tatsache, dass sich nur rund 15\% der Ablehnungsgründe auf negative Bewertungen der Marktchancen der Innovationen (3\%) bzw. negative Bewertungen des beantragenden Unternehmens (7\%) oder des EFS (5\%) bezogen, während der Großteil der Ablehnungen auf (grundsätzliche) materielle (keine Feasibility, wirtschaftliche Frage im Vordergrund, keine Innovation) und formelle Mängel der Anträge entfällt, ist davon auszugehen, dass seitens des FFF nicht versucht wurde, prima vista eventuell unrealistische - Ideen bereits in der Beantragungsphase zu stoppen. Dies spricht einerseits für die Qualität der eingebrachten FeasibilityVorschläge und andererseits für ein unvoreingenommenes und damit innovationsfreundliches Förderungsverhalten des FFF.

Bezüglich eines weiteren Kriteriums zur Messung der Professionalität im Start der Innovationsprojekte, nämlich der Korrelation zwischen der technischen Bewertung der FS und der Beantragung von Folgeprojekten, hat bereits die Auswertung in Punkt I./6. (vgl. S. 13) gezeigt, dass kein dementsprechender Zusammenhang nachgewiesen werden kann. 


\section{Anregung weiterer Projekte / Kooperationen}

Die AFS hat weitere Projekte und Kooperationen induziert:

- Die Unternehmen planen - soweit die Abschlussberichte vorliegen - in überwiegendem Ausmaß die Weiterführung des FS-Projekts.

- Sie wollen auch die Kooperation mit dem EFS fortführen

- und würden bei neuen Fragestellungen wiederum eine Feasibility Study beauftragen.

- Jeder 5. geförderte AFS-Antrag zieht einen weiterführenden Folgeantrag nach sich, von denen 3/4 (bzw. bei positiver Erledigung der offenen Anträge fast 9/10) auch gefördert werden können.

- Zwar sind die in der AFS geförderten Anträgen und die nicht geförderten Anträge in etwa mit der gleichen Zahl an nachfolgenden Anträgen (57\%, $59 \%$ ) verbunden, allerdings mobilisieren geförderte Anträge wesentlich mehr Unternehmen, weitere FFF-Anträge einzubringen. (In der Gruppe der nicht geförderten AFS-Anträge befinden sich 4 Antragsteller, die zusammen 22 weitere FFF-Anträge stellten und so die Quote verzerren.)

\begin{tabular}{|c|c|c|c|}
\hline subjektive Einsc & $\begin{array}{c}\text { רätzung der Weiterfüh } \\
\text { Mittelwert (1=ja sicher } \\
\text {...4=nein) }\end{array}$ & Anteil ,ja sicher" & Anteil „eher ja“ \\
\hline $\begin{array}{l}\text { planen Weiterfüh- } \\
\text { rung des Projekts } \\
\text { (FB Fr. 7) }\end{array}$ & 1,5 & $66 \%$ & $22 \%$ \\
\hline subjektive Einsc & $\begin{array}{c}\text { hätzung der weiteren } \\
\text { Mittelwert (1=ja sicher } \\
\ldots 4=\text { nein })\end{array}$ & $\begin{array}{l}\text { Oooperation mit EFS } \\
\text { Anteil „ja sicher" }\end{array}$ & Anteil „eher ja“ \\
\hline $\begin{array}{l}\text { planen weitere } \\
\text { Kooperationen mit } \\
\text { EFS (FB Fr. 8) }\end{array}$ & 1,3 & $80 \%$ & $14 \%$ \\
\hline subjektive Einsc & $\begin{array}{c}\text { hätzung der neuerliche } \\
\text { Mittelwert (1=ja sicher } \\
\ldots 4=\text { =inein) }\end{array}$ & $\begin{array}{c}\text { n Beauftragung mit FS } \\
\text { Anteil ,ja sicher" }\end{array}$ & $\begin{array}{l}\text { oi neuem Thema } \\
\text { Anteil „eher ja“ }\end{array}$ \\
\hline $\begin{array}{l}\text { würden wiederum } \\
\text { FS beauftragen } \\
\text { (FB Fr. 9) }\end{array}$ & 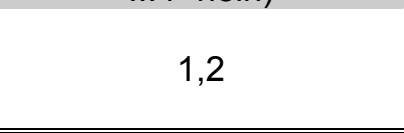 & $89 \%$ & $7 \%$ \\
\hline tatsächliche Fols & $\begin{array}{l}\text { eprojekte (hier konnten } \\
\text { geförderte AFS-Anträge } \\
\text { im für Folgeanträge } \\
\text { relevanten Zeitraum }\end{array}$ & $\begin{array}{l}\text { laturgemäß nur FFF-Anträg } \\
\text { weiterführende } \\
\text { Folgeanträge }\end{array}$ & $\begin{array}{l}\text { erücksichtigt werden) } \\
\text { geförderte (bzw. } \\
\text { geförderte oder noch } \\
\text { nicht entschiedene) } \\
\text { Folgeanträge }\end{array}$ \\
\hline $\begin{array}{l}\mathrm{n} \\
\%\end{array}$ & 87 & $\begin{array}{c}18 \\
20,5 \% \text { Folgeprojektquote }\end{array}$ & $\begin{array}{c}14(16) \\
16(18,5) \text { Quote der } \\
\text { erfolgreichen } \\
\text { Folgeanträge }\end{array}$ \\
\hline \multicolumn{4}{|c|}{ tatsächliche weitere FFF-Projekte (nicht nur die die FS weiterführenden Projekte) } \\
\hline $\begin{array}{l}\text { geförderte AFS- } \\
\text { Anträge }\end{array}$ & $\begin{array}{l}125 \text { geförderte AFS- } \\
\text { Anträge im } \\
\text { Evaluierungszeitraum }\end{array}$ & \begin{tabular}{|c|}
71 weitere nachfolgende \\
FFF-Anträge von 44 \\
Unternehmen $(\varnothing 1,6$ \\
Anträge/Unternehmen) \\
\end{tabular} & $\begin{array}{c}57 \% \text { Antragsquote } \\
35 \% \text { Wieder- } \\
\text { beantragungsquote }\end{array}$ \\
\hline $\begin{array}{l}\text { nicht geföderte } \\
\text { AFS-Anträge }\end{array}$ & $\begin{array}{l}58 \text { nicht geförderte AFS- } \\
\text { Anträge im } \\
\text { Evaluierungszeitraum }\end{array}$ & $\begin{array}{l}34 \text { weitere nachfolgende } \\
\text { FFF-Anträge von } 14 \\
\text { Unternehmen }(\varnothing 2,4 \\
\text { Anträge/Unternehmen) }\end{array}$ & $\begin{array}{l}59 \% \text { Antragsquote } \\
24 \% \text { Wieder- } \\
\text { beantragungsquote }\end{array}$ \\
\hline
\end{tabular}

Tabelle 15 


\section{Akquisition qualitativ hochwertiger Folgeprojekte}

Von den 20 bisher eingegangenen Folgeanträgen wurden 15 gefördert, 2 abgelehnt und bei drei Anträgen steht die Entscheidung noch aus. Daraus ergibt sich eine Ablehnungsquote von $12 \%$ (2 von 17).

Angesichts dieser geringen Ablehnungsquote bei Folgeprojekten kann wohl geschlossen werden, dass der FFF über die AFS qualitativ hochwertige Folgeanträge akquirieren konnte.

Eine höhere Qualität der Folgeprojekte konnte anhand der P-, T-, F- und W-Werte nicht festgestellt werden. Der Vergleich zwischen diesen Parametern der Folgeprojekte und der vom FFF ermittelten 3-Jahres-Durchschnitte der klein- und mittelbetrieblichen Anträge zeigt keine deutlichen Abweichungen. Auch die Differenzen zwischen diesen Parametern und dem jeweiligen Jahresdurchschnitt aller KMU-Anträge bestätigen dieses Bild.

\begin{tabular}{|c|c|c|}
\hline & Folgeprojekte & KMU-Durchschnitt (2000-03) \\
\hline \multirow[t]{2}{*}{ P-Wert } & 30 & 32,3 \\
\hline & $\begin{array}{ll}\varnothing & \text { Differenz der P-Werte der } \\
\text { Jahresdurchschnitte der KMU bezo }\end{array}$ & $\begin{array}{l}\text { Folgeprojekte und der jeweiligen } \\
\text { ogenen P-Werte: }-2,1\end{array}$ \\
\hline \multirow[t]{2}{*}{ T-Wert } & 28,7 & 31,6 \\
\hline & $\begin{array}{l}\varnothing \text { Differenz der T-Werte der } \\
\text { Jahresdurchschnitte der KMU bezo }\end{array}$ & $\begin{array}{l}\text { Folgeprojekte und der jeweiligen } \\
\text { ggenen T-Werte: }-2,6\end{array}$ \\
\hline \multirow[t]{2}{*}{ F-Wert } & \begin{tabular}{|c|}
24,5 \\
\end{tabular} & 25,4 \\
\hline & $\begin{array}{l}\varnothing \text { Differenz der F-Werte der } \\
\text { Jahresdurchschnitte der KMU bezo }\end{array}$ & $\begin{array}{l}\text { Folgeprojekte und der jeweiligen } \\
\text { ggenen F-Werte: }-0,5\end{array}$ \\
\hline \multirow[t]{2}{*}{ W-Wert } & \begin{tabular}{|c|}
26,8 \\
\end{tabular} & 29,0 \\
\hline & $\begin{array}{l}\varnothing \text { Differenz der W-Werte der } \\
\text { Jahresdurchschnitte der KMU bezo }\end{array}$ & $\begin{array}{l}\text { Folgeprojekte und der jeweiligen } \\
\text { ggenen W-Werte: }-2,1\end{array}$ \\
\hline
\end{tabular}

Tabelle 16

\section{Verbesserung der wirtschaftlichen Laqe der geförderten Unternehmen}

Da hierzu Angaben wie das durch das Innovationsvorhaben erwartete Umsatz- und Exportwachstum, etc. nicht verfügbar sind, kann hier nur die Frage geprüft werden, ob sich das Projekt in Richtung Umsetzung entwickelt und welches Förderungsvolumen die geförderten Unternehmen bei den nachfolgenden Umsetzungsschritten lukrieren konnten. Diesen Indikatoren liegen folgende Überlegungen zugrunde:

- Wenn ein innovatives Projekt umgesetzt wird, steigen die Marktchancen und/oder die Wettbewerbsstärke eines Unternehmens.

- Wenn Teile der Umsetzungskosten durch öffentliche Förderungen abgedeckt werden können, hat das positive Auswirkungen auf die Liquiditäts- und Ertragssituation des Unternehmens.

Die Auswertung der Frage 7 aus dem Fragebogen („Beabsichtigen Sie aufgrund der Ergebnisse die Durchführung eines weiterführenden Projekts?") ergab folgende Ergebnisse: 
56 Unternehmen (65,9\%) antworteten mit „ja“ (Wert 1), 19 Unternehmen mit „eher ja“ (Wert 2), 7 Unternehmen mit "eher nein“ (Wert 3) und 3 Unternehmen mit "nein" (Wert 4). Es ergab sich ein Mittelwert von 1,49, womit zum Zeitpunkt der Schlussberichterstattung ein Großteil der Unternehmen an eine Fortführung des mittels der FS initiierten Projektes dachte.

Bezüglich der effektiven Umsetzung von Folgeprojekten in Form von FFF-Folgeprojektanträgen ergibt sich folgendes Bild:

Die 15 geförderten Folgeprojekte erreichten ein Gesamtförderungsvolumen seitens des FFF von $€$ 589.823,- (Mittelwert $€$ 39.322,-). Da sich der Gesamtbetrag der Feasibility-Förderungen dieser 15 Projekte auf $€$ 154.356,- (Mittelwert $€ 10.290,-$ ) belief, kann man schlussfolgern, dass in Bezug auf diese 15 Feasibilities, die zu geförderten Folgeprojekten führten, aus jedem EURO an Feasibility-Förderung 3,82 $€$ Folgeprojektförderung wurden.

Insgesamt bedeuten diese 15 Folgeprojekte ein Investitionsvolumen von $€$ 2,615.000,- (Mittelwert $€$ 174.350,-). In Bezug auf diese 15 Feasibilities induzierte jeder EURO an Feasibility-Förderung somit fast $€$ 17,- an Folgeinvestitionen.

Bezieht man die Fördervolumina der Folgeprojekte auf die Summe der vor dem 30.06.02 (siehe Tabelle 9) - dem für Folgeprojekte relevanten Zeitraum - gewährten Feasibility-Förderungen ( $€$ 697.288,-), so führte jeder EURO Feasibility Förderung zu $0,85 €$ Folgeprojektförderung und zu 3,6 $€$ (bzw. unter Einbeziehung der noch offenen Entscheidungen zu Folgeprojekten) 4,2€ gesamten Folgeinvestitionen. 


\section{MODUL II: EFFIZIENZ DER AKTIONSLINIE „FEASIBILITY STUDIES“}

1. Kundenorientierung

\begin{tabular}{|c|c|c|c|c|}
\hline \multirow[t]{2}{*}{ Ereignis } & \multirow[b]{2}{*}{$n$} & \multicolumn{3}{|c|}{$\begin{array}{c}\text { zeitlicher Abstand zum jeweils vorgelagerten } \\
\text { Ereignis in Tagen }\end{array}$} \\
\hline & & Mitte/wert & Minimum & Maximum \\
\hline \multicolumn{5}{|l|}{\begin{tabular}{l|l|} 
Eingangsdatum des Antrags & \\
zur Feasibility Study
\end{tabular}} \\
\hline Mitteilungsdatum & 173 & 53,6 & 22 & 154 \\
\hline Übereinkommen retour & 113 & 17,4 & 2 & 100 \\
\hline 1. Rate & 115 & 9,1 & 1 & 30 \\
\hline Endbericht & 89 & 166,6 & -12 & 532 \\
\hline 2. Rate & 75 & 68,7 & 12 & 385 \\
\hline $\begin{array}{l}\text { Gesamtabwicklung (Eingang } \\
\text { bis 2. Rate) }\end{array}$ & 75 & 320,7 & 91 & 603 \\
\hline
\end{tabular}

Tabelle 17

Das Ziel, die Anträge innerhalb von 6 Wo. zu erledigen (vom Antragseingang bis zur Mitteilung), wurde im Durchschnitt knapp verfehlt, in 38\% der Anträge hingegen erreicht!

Die Auszahlungen erfolgen prompt. Verzögerungen liegen zumeinst in der Verantwortung der Antragsteller (Differenz zwischen Mitteilungsdatum und Rücksendung der Übereinkommen) bzw. sind in der Durchführungsdauer der FS begründet (Differenz zwischen Mitteilungsdatum und Übermittlung des Endberichts).

Die zeitliche Abfolge (z.B. Endbericht vor der ersten Rate) bzw. Einreichung des Folgeprojekts vor Übermittlung des Endberichts sind ein Indiz dafür, dass hier eventuell auch Feasibility Studien gefördert wurden, die de facto vor Genehmigung durch den FFF bereits durchgeführt und nachträglich unter der AFS eingereicht wurden. (Da den Antragstellern in diesem Fall die Ergebnisse bereits bekannt waren, konnten auch Folgeprojekte vor dem Vorliegen der "offiziellen Endberichte" gestartet werden.)

Die durchschnittliche Gesamtdauer der FS scheint aber angesichts der oft kurzen windows of opportunity für einen Markteintritt und dem Umstand, dass es sich dabei um eine grundsätzliche technische Machbarkeitsanalyse handelt, tendenziell verbesserungsfähig.

Die Zufriedenheit mit der Abwicklung durch den FFF fällt äußerst positiv aus (85\% bewerten die Abwicklung durch den FFF mit sehr gut, 15\% mit gut, $\varnothing 1,16$ ). Formulierungen wie „überraschend unkompliziert", „erstaunlich unbürokratisch“ etc. zeigen auch die Differenz zur Erwartungshaltung gegenüber Förderungsinstitutionen. Wobei die Bewertung von jenen Unternehmen, die zum ersten Mal mit dem FFF zu tun hatten, besonders gut ausfällt $(\varnothing 1,14$ im Vergleich zu $\varnothing 1,18$ bei den „Stammkunden“). Dennoch deuten vereinzelte Hinweise der Stammkunden an, dass sich die Abwicklung der AFS auch im Vergleich zu anderen FFF-Programmen positiv abhebt. 
Zusätzlich nimmt die Zufriedenheit mit der Abwicklung durch den FFF leicht zu (Anträge 1999: $\varnothing 1,2$; Anträge 2000: $\varnothing 1,25$; Anträge 2001: $\varnothing 1,12$; Anträge 2002: $\varnothing$ 1,11). Das zeigt sich auch in der laufenden Beschleunigung der Antragserledigung durch den FFF:

\begin{tabular}{|l|c|c|c|c|c|}
\hline \multirow{2}{*}{ Ereignis } & \multicolumn{5}{|c|}{$\begin{array}{c}\text { zeitlicher Abstand zum jeweils } \\
\text { vorgelagerten Ereignis in Tagen } \\
\text { Mittelwerte der Anträge aus den Jahren }\end{array}$} \\
\cline { 2 - 6 } & 1999 & 2000 & 2001 & 2002 & 2003 \\
\hline Eingangsdatum des Antrags zur AFS & & & & & \\
\hline Mitteilungsdatum & - & 53,4 & 59,2 & 53,3 & 43,7 \\
\hline Übereinkommen retour & - & 16,7 & 22 & 13,4 & 20,4 \\
\hline 1. Rate & - & 8,5 & 11,4 & 8,8 & 7,6 \\
\hline Endbericht & - & 216,5 & 151,2 & 149,2 & - \\
\hline 2. Rate & - & 79,9 & 75,1 & 54,1 & - \\
\hline $\begin{array}{l}\text { Gesamtabwicklung (Eingang bis 2. Rate) } \\
\text { „-“...aufgrund der geringen Fallzahl keine interpretierbaren Resultate }\end{array}$ & - & 373,3 & 330,1 & 266,7 & - \\
\hline
\end{tabular}

Tabelle 18

Im Jahr 2003 hat der FFF sein selbstgestecktes Ziel, Anträge innerhalb von 6 Wochen zu erledigen, im Durchschnitt fast erreicht.

\section{Effizienz des Mitteleinsatzes}

Die Frage nach der Effizienz des Mitteleinsatzes soll mittels der Analyse zweier Fragen aus dem FB, nämlich jener nach der Möglichkeit des Erreichens der Ergebnisse auf anderem Wege (FB Fr. 3) sowie des Preis-Leistungs-Verhältnisses der FS (FB Fr. 4) vorgenommen werden.

In Bezug auf die Frage nach der Erreichbarkeit des Ergebnisses auf anderem Wege ergaben sich die folgenden Antworthäufigkeiten:

\begin{tabular}{|lc|c|c|c|}
\cline { 3 - 5 } \multicolumn{1}{c|}{} & Häufigkeit & gültige Prozent & $\begin{array}{c}\text { kumulierte } \\
\text { Prozente }\end{array}$ \\
\hline ja & $(1)$ & 3 & 3,5 & 3,5 \\
\hline eher ja & $(2)$ & 9 & 10,6 & 14,1 \\
\hline eher nein & $(3)$ & 26 & 30,6 & 44,7 \\
\hline nein & $(4)$ & 47 & 55,3 & 100,0 \\
\hline gesamt & 85 & 100,0 & \\
\hline
\end{tabular}

Lediglich 14\% der Antwortenden waren der Anschauung, dass die Ergebnisse auch auf anderem Weg erreicht hätten werden können (3,5\% „ja“; 10,6\% „eher ja“), während rund $86 \%$ der Antwortenden die FS als einzigen Weg zu Erreichung der erzielten Ergebnisse sahen. Der Mittelwert der Nennungen liegt bei 3,38 (zwischen "eher nein" und „nein“)

Ein ebenso positives Bild im Sinne der Effizienz der AFS zeigen die Beurteilungen des Preis-Leistungs-Verhältnisses der FS durch die Befragten: 


\begin{tabular}{|lr|r|r|r|}
\cline { 2 - 5 } \multicolumn{1}{c|}{} & \multicolumn{2}{|c|}{ Häufigkeit } & gültige Prozente & $\begin{array}{c}\text { kumulierte } \\
\text { Prozente }\end{array}$ \\
\hline sehr gut & $(1)$ & 26 & 30,6 & 30,6 \\
\hline gut & $(2)$ & 47 & 55,3 & 85,9 \\
\hline schlecht & 10 & 11,8 & 97,6 \\
\hline sehr schlecht (4) & 2 & 2,4 & 100,0 \\
\hline gesamt & \multicolumn{2}{|r|}{85} & 100,0 & \\
\hline
\end{tabular}

Rund $86 \%$ der Antwortenden beurteilten das Preis-Leistungs-Verhältnis der FS als "sehr gut" $(30,6 \%)$ oder „gut“ (55,3\%). Die restlichen rund $14 \%$ waren mit dem PreisLeistungs-Verhältnis der EFS weniger zufrieden und beurteilten es als "schlecht“ $(11,8 \%)$ oder sogar als „sehr schlecht“ $(2,4 \%)$. Somit ergibt sich ein Mittelwert von 1,86 (zwischen „sehr gut" und „gut"). 


\section{Gesamtresümee der Zwischenevaluierung der AFS}

Mit der Aktionslinie wurden mit wenigen Einschränkungen die gesteckten Ziele erreicht.

- Kritisch muss angemerkt werden, dass ein vermuteter und angestrebter positiver Zusammenhang zwischen den Ergebnissen der Feasibility Studie und der Wahrscheinlichkeit der Beantragung von weiterführenden Projekten nicht nachgewiesen werden konnte. Allerdings kann die mangelnde Nachweisbarkeit der Anreizwirkung positiver Feasibility Studien bzw. der Signalwirkung negativer FS auch auf die geringe Aussagekraft der dieser Auswertung zugrundeliegenden Kennzahl „technische Bewertung Erfolg“ zurückgeführt werden (siehe dazu nachfolgende Empfehlung zur Verbesserung der Evaluierungsmöglichkeiten).

- Das Ziel, Anträge innerhalb von 6 Wochen zu erledigen, wurde knapp verfehlt, wenn auch Verbesserungen im Laufe der AFS festgestellt werden können.

- Mit der AFS wurden professionelle und qualitativ hochwertige Feasibility Studies mit einem guten Preis-Leistungs-Verhältnis induziert.

- Die Zusammenarbeit der Unternehmen mit dem Ersteller der Feasibility Studie mündet in vielen Fällen in langfristigen Kooperationen, was als Impuls für die Etablierung von technischen Beratungsnetzwerken gewertet werden kann.

- Mit den Förderungen wurden Feasibility Studien angeregt, die ein erhebliches Geldvolumen ( $€$ 1,5 Mio. Förderungen und Eigenleistungen) bewegt haben, sodass insgesamt von einem wesentlichen Impuls für die österreichische Forschungslandschaft gesprochen werden kann, von dem eine große Zahl (= 85) von sehr heterogenen Forschungsinstitutionen (private Unternehmen, Universitäten, Institute von Branchenverbänden, etc.) profitierte.

- Dem FFF ist es mit der AFS gelungen, Gründungs- und Jungunternehmen anzusprechen und zu motivieren, das Projekt fortzusetzen. Diese Unternehmen lernen somit frühzeitig, professionelle Unterstützungsnetzwerke (Berater, Förderungsinstitutionen, etc.) zu nutzen.

- Da Folgeprojekte erst mit einem etwa einjährigen time-lag beantragt werden, kann das Gesamtvolumen der induzierten Folgeprojekte noch nicht vollständig erfasst werden. Aus der Analyse der zu diesem Zweck definierten Teilkohorte kann aber doch der Schluss gezogen werden, dass Feasibility Studien zu einem hohen Anteil (16\%-19\%) in Folgeprojekten münden und jeder EURO Förderung im Rahmen der AFS einen erheblichen Forschungsmultiplikator (3,6-4,2) aufweist. (Da diese Evaluierung auf FFF-geförderte Folgeprojekte beschränkt war, werden hier die entsprechenden Zahlen systematisch unterschätzt.)

- Auch wurden bisher offenbar nicht oder nicht professionell innovierende Unternehmen motiviert, Innovationsideen tatsächlich umzusetzen und/oder im Vorfeld ihre Umsetzbarkeit professionell zu prüfen. Insofern geht von der Aktionslinie auch ein Impuls zur Veränderung der Einstellungen der österreichischen KMUs aus.

- Diese Einstellungsänderung dürfte nicht nur das Innovationsverhalten sondern auch die Einstellungen gegenüber technischen/wissenschaftlichen Beratern betreffen, waren doch die geförderten KMUs in ihrer Mehrzahl bezüglich Qualität 
und Nutzbarkeit der Studien positiv überrascht. Es ist zu vermuten, dass so die psychische Distanz und die damit einhergehenden „Berührungsängste“ zwischen Unternehmen und z.B. universitären Einrichtungen reduziert werden konnten.

- Die Einstellungsänderungen betreffen aber auch Förderungsinstitutionen: Dem FFF ist es durch die Art der Abwicklung der Aktionslinie gelungen, KMUs - die gegenüber Förderungsinstitutionen grundsätzlich skeptisch sind - geradezu zu verblüffen, werden doch die Bewertungen des FFF durch Aussagen wie „überraschend unbürokratisch und hilfreich, etc.“ dominiert. 


\section{Empfehlungen}

\section{Empfehlungen zur Verbessung der Evaluierungsmöglichkeiten}

- Verbesserung des Fragebogens:

- Bewertung der Ergebnisse der Feasibility Study: Die Frage 6 des Fragebogens des Abschlussberichts wurde teilweise missverstanden: Die Kommentare zeigen, dass die Antworten zu dieser Frage sowohl auf die Qualität der Ergebnisse als auch auf den Inhalt der Ergebnisse Bezug nehmen (z.B. „Habe eine professionelle Arbeit erwartet!“ $\rightarrow$ Erwartungen erfüllt; „Hoffte auf neue Erkenntnisse“ $\rightarrow$ Erwartungen nicht erfüllt; „Habe negatives Resultat erwartet, es war überraschend positiv" $\rightarrow$ Erwartungen nicht erfüllt). Da bei knappen Antworten die Intention des Befragten nicht erkennbar ist, kann dieses Problem auch nicht nachträglich korrigiert werden.

Hier sollte die Frage präziser gefasst und die beiden Interpretationsmöglichkeiten in zwei Fragestellungen getrennt werden (die Professionalität der FS wird ohnehin in Frage 5 abgefragt), also etwa:

a) „Wir haben uns sehr wenige, wenige, viele bzw. sehr viele neue Erkenntnisse erwartet. “ - „Die FS hat sehr wenige, wenige, viele bzw. sehr viele neue Erkenntnisse gebracht."

b) „Wir haben eine deutlich positive, eine positive, eine negative bzw. eine deutlich negative technische Bewertung erwartet.“ - „Das Ergebnis der FS brachte eine deutlich positive, eine positive, eine negative bzw. eine deutlich negative Bewertung zu Tage."

- Vergleichseinstellungen: Interessant ist die durch eine solche Aktionslinie induzierte Einstellungsänderung. Zur fundierteren Beleuchtung dieses Aspekts müssten aber Einstellungsdaten (Werte, Erwartungen, etc.) zum Zeitpunkt der Antragstellung vorliegen. Da hier die Gefahr besteht, dass die Antragsteller vermeintlich erwünschtes Antwortverhalten zeigen, sollten diese Fragebögen gar nicht an den FFF, sondern bei der Antragstellung bereits an die evaluierende Institution geschickt und dort gesammelt werden.

- Innovationsverhalten: Inwieweit tatsächlich die Basis innovierender Unternehmen vergrößert werden konnte, konnte nicht eingehend überprüft werden. Hier musste unterstellt werden, dass jene Unternehmen, die beim FFF Neukunden sind, zuvor nicht zur Gruppe der „innovativen Unternehmen“ gehörten. Eine Abfrage des vergangenen Innovationsverhaltens (zum ersten Mal eine technische FS, zum ersten Mal eventuell ein größerer Innovationsschub mit FS verbunden, etc.) könnte hier die Evaluierungsmöglichkeiten verbessern.

- technische Bewertung: Für die Bewertung des FS lag nur die Kennzahl „technische Bewertung Erfolg“ vor, die sich aus der Bewertung „Qualität und Erfolg“ sowie „Verwertung“ zusammensetzt. Korrelationen zwischen diesen Teilbewertungen als positive bzw. negative Impulse zur Projektfortführung und der Wahrscheinlichkeit von Folgeprojekten konnten so nicht isoliert analysiert werden. Zwischen der Gesamtbewertung und den Folgeprojekten konnte keine 
positive Korrelation nachgewiesen werden. Es kann aber nicht ausgeschlossen werden, dass teilweise gegenläufige Teilbewertungen (z.B. positive technische Bewertung bei kritischen Verwertungsaspekten) entsprechende Zusammenhänge verstellen, womit eventuell wichtige Effektivitätsnachweise der AFS verdeckt werden. Zur Lösung dieses Problems wird vorgeschlagen, die Unternehmer bezüglich ihrer Einschätzung des Ergebnisses der Feasibility („klare GO-Entscheidung“ .... „klare STOP-Entscheidung“) zu befragen (siehe oben).

- Detailempfehlungen zur Datenerfassung:

- Datenbankerfassung der Zahl der unselbständig Beschäftigten aus dem Antrag: Damit könnte man auf den zur Antragstellung aktuellen Wert zugreifen (teilweise erhebliche Abweichungen $\mathrm{zu}$ den Daten aus den letzten 3 Bilanzjahren).

- Datenbankerfassung der EFS mit gleichem Wortlaut bzw. codiert: Damit wäre die Evaluierung mit weniger Aufwand verbunden, so musste seitens des FFF diese Information aus den einzelnen Anträgen herausgefiltert werden.

- Datenbankerfassung der geschaffenen F\&E-Arbeitsplätze: Diese Erfassung ist zwar bei der AFS weniger relevant (durch eine FS werden wohl kaum F\&EArbeitsplätze geschaffen), für die Bewertung der Folgeprojekte und damit der Effekte der AFS erscheint diese Information aber sehr wohl relevant.

\section{Empfehlungen zur Verbessung der AFS}

- Vermeidung von Missverständnissen: Da ca. die Hälfte der negativ beschiedenen Anträge abgelehnt wurde, weil die Anträge nicht auf die Durchführung von technischen Machbarkeitsstudien hinausliefen, sollte die Präsentation der Richtlinie überdacht werden. Klarere Formulierungen, Beispiele auch für nichtgeförderte Anliegen, etc. könnten die Effizienz der AFS (weniger Bewertungsaufwand beim FFF) erhöhen.

- „Kleine“ Feasibility Studien haben eine geringere Zieleffektivität (geringere Wahrscheinlichkeit, dass sie in einem Folgeprojekt münden). Daher könnte einerseits - auch im Interesse der Effizienz der Administration der Fördermittel eine Untergrenze des Förderungsbeitrages je FS angedacht werden. Andererseits ist zu bedenken, dass eine solche Untergrenze ein negatives Signal für kleinere Betriebe bedeuten könnte.

- Die Effizienz des FFF in der Abwicklung der AFS steigt laufend (kürzere Abwicklungszeiten, höhere Zufriedenheiten der Antragsteller). Aktionslinien sollten vor diesem Hintergrund grundsätzlich über einen längeren Zeitraum laufen.

- Zur Beschleunigung der FS und damit zur Verkürzung der „time to market“ könnten Modifikationen der Richtlinien (z.B. Prämien bzw. Abschläge in Abhängigkeit der Zeitdauer) angedacht werden, da Verzögerungen im wesentlichen die Antragsteller und die EFS zu verantworten haben. 


\section{Gesamtbewertung}

Da mit der AFS

- Unternehmen an ein professionelles Innovationsmanagement herangeführt werden, indem in einem hohen Ausmaß Unternehmen angesprochen werden, die bisher das Angebot des FFF nicht nutzten,

- ein breites Spektrum an technischen Beratungsinstitutionen in die Innovationsvorhaben eingebunden wurde,

- in hohem Maße Folgeprojekte initiiert wurden

- und insgesamt ein großes Volumen an Forschungsaufwendungen mobilisiert wurde

empfehlen wir die Weiterführung dieser Aktionslinie.

Die weitere Entwicklung der Aktionslinie sollte u.E. anhand folgender Relationen beobachtet werden:

- Veränderung des Anteils der Anträge von Neukunden an den gesamten Anträgen im Zeitablauf

- Veränderung des Anteils der Anträge von Antragstellern, die bereits früher in der Aktionslinie „Feasibility Studies" Förderungen erhalten haben, an den gesamten Anträgen im Zeitablauf

Bei starkem Ansteigen dieser beiden Kennzahlen ist u.E. die Weiterführung der Aktionslinie zu hinterfragen. 